\title{
EVIDENCE FOR TRAPPED ANOMALOUS COSMIC RAY OXYGEN IONS IN THE INNER MAGNETOSPHERE
}

\author{
N.L. Grigorov', M.A. Kondratyeva', M.I. Panasyuk ${ }^{1}$, Ch.A. Tretyakova 1 , \\ J.H. Adams, Jr. ${ }^{2}$, J.B. Blake ${ }^{3}$, M. Schulz ${ }^{3}$, R.A. Mewaldt ${ }^{4}$, A.J. Tylka ${ }^{5}$
}

Abstract, A series of measurements of 5-30 $\mathrm{MeV} /$ nucleon oxygen ions made with track detector stacks on Cosmos satellites show isotropic angular distributions during solar energetic particle events. Solar-quiet times, on the other hand, have highly anisotropic distributions suggestive of a trapped-particle component. Detailed Monte Carlo simulations confirm this interpretation and allow us to measure the trapped and cosmic-ray contributions to the observed fluxes. Our data are fully consistent with anomalous cosmic-ray ions, rather than radial diffusion from the outer zone, as the source of the trapped particles.

\section{Introduction}

There have been several reports of excess oxygen flux at energies $\sim 10 \mathrm{MeV} /$ nucleon in the inner magnetosphere [Mogro-Campero and Simpson, 1970; Chan and Price, 1975; Biswas and Durgaprasad, 1980; Oschlies et al., 1989; Adams et al., 199/b]. These observations, however, were unable to establish the nature of this flux and, in particular, to prove that the excess ions followed trapped-particle trajectories. If trapped, such ions may yield insights into magnetospheric processes and may also be of astrophysical interest. The anomalous component of cosmic rays [reviewed by Webber, 1989] comprises primarily $\mathrm{He}, \mathrm{N}, \mathrm{O}$, and $\mathrm{Ne}$ atoms from the local neutral interstellar medium which have entered the heliosphere, become singly-ionized by the solar wind or solar UV [Fisk et al., 1974], and then been accelerated to energies $\gtrsim 10 \mathrm{MeV} /$ nucleon, probably at the solar-wind termination shock [Pesses et al., 1981]. Blake and Friesen [1977] noted that such particles could penetrate deeply into the magnetosphere and become geomagnetically trapped after being stripped of remaining electrons in the residual atmosphere, with subsequent lifetimes ranging from hours to months. In this case, these trapped particles are a sample of interstellar matter directly available for study at Earth.

In this Letter we report on a series of observations of 5$30 \mathrm{MeV} /$ nucleon oxygen ions in the inner magnetosphere in 1986-89 [cf. Grigorov et al., 1989 and references therein]. The observations were made with small cellulose nitrate

\footnotetext{
${ }^{1}$ Institute for Nuclear Physics, Moscow State University

${ }^{2}$ E.O. Hulburt Center for Space Research, Naval Research Laboratory

${ }^{3}$ Space and Environment Technology Center, The Aerospace Corporation

${ }^{4}$ California Institute of Technology

$5_{\text {Universities Space Research Association }}$
}

Copyright 1991 by the American Geophysical Union.

Paper number 91GL02551

0094-8534/91/91GL-02551 \$03.00 detector stacks flown for $\sim 14$ days on Cosmos satellites in nearly circular orbits at altitudes of $250-400 \mathrm{~km}$ and inclinations of $62^{\circ}-82^{\circ}$. The detectors registered and identified CNO ions. Lighter species (such as $\mathrm{He}$ ) did not leave tracks in the detectors, and heavier species could not be identified. Although the detectors recorded no timing information for individual nuclei, the angular distributions of the tracks clearly showed the presence of trapped particles. Since the spacecraft were three-axis stabilized, the detectors always passed through the low-altitude radiation belts with the same attitude. Trapped particles thus arrived from characteristic directions and registered at characteristic angles. Data from these same flights, with angular cuts to exclude trapped particles, have previously been combined with simultaneous measurements outside the magnetosphere to demonstrate conclusively that anomalous cosmic-ray oxygen ions are singly ionized [Adams et al., 1991a].

\section{Observations}

The dip angle $\theta$ and azimuth angle $\phi$, as defined in Figure 1, specify a particle's arrival direction in the detector coordinate system. Figure 2 shows the observed arrival directions for particles collected in two typical Cosmos exposures, one when solar energetic particles (SEPs) dominated the fluxes and one during quiet-time, as identified by Caltech proton and He measurements on IMP-8 in interplanetary space [cf. Adams et al., 1991a]. During the SEP-dominated period, the particles arrived from around the

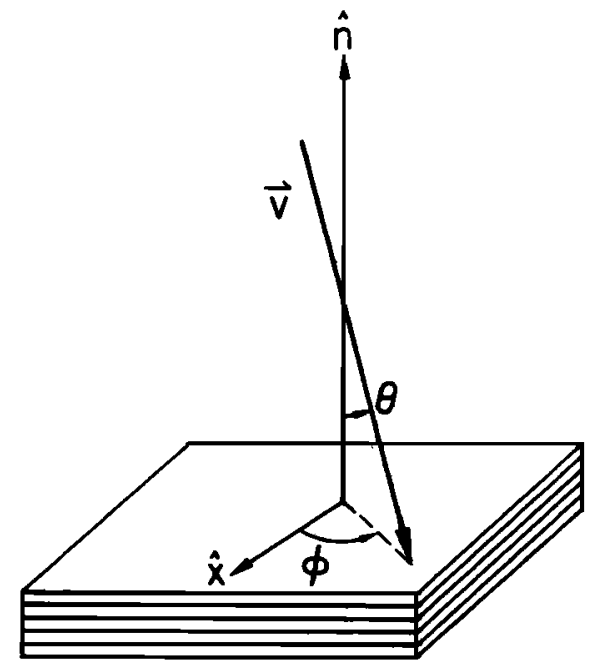

Fig. 1. Arrival-direction angles in the detector coordinate system. Dip angle $\theta$ is the angle between the particle's velocity vector $\mathbf{v}$ and the normal to the detector, $n$. Azimuth angle $\phi$ is the angle between the projection of $v$ onto the detector surface and a fixed reference direction $x$ in the detector plane. 

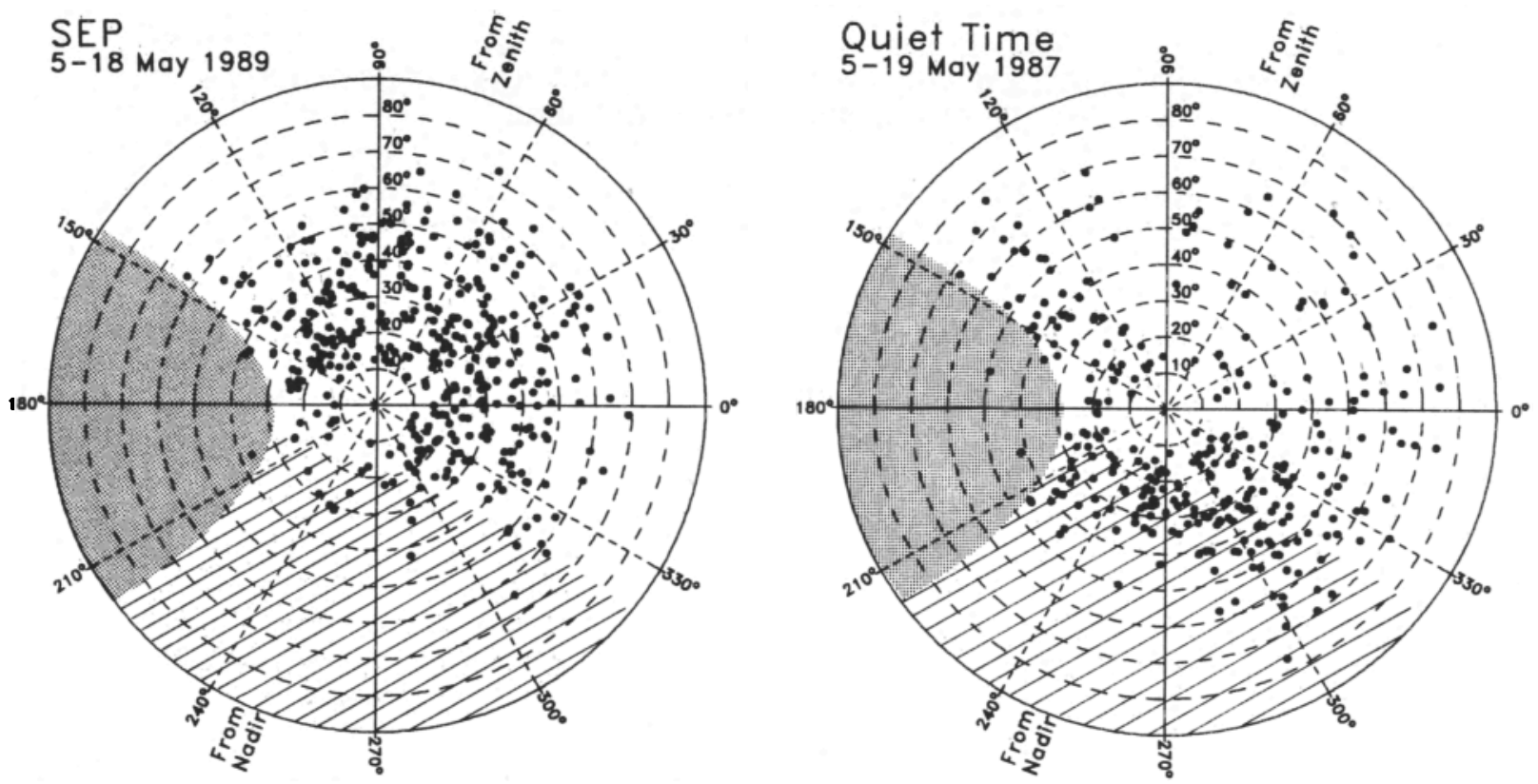

Fig. 2. Polar diagrams of observed arrival directions in a SEP-dominated exposure (left) and in a quiet-time exposure (right). Each point represents a single particle, with $\theta$ and $\phi$ plotted as the radial and azimuthal coordinates, respectively. The zenith and nadir directions are noted. Shaded areas show arrival directions blocked by obstructions on the satellite. The hatched area shows arrival directions which are below the Earth's horizon. Tracks at $\theta<10^{\circ}$ and $\theta>80^{\circ}$ are omitted due to detection inefficiencies.

zenith direction in a distribution which was isotropic, except for shadows caused by obstructions on the satellite and the solid Earth. During quiet-time, however, there were relatively few particles from around the zenith direction. A large number of particles came from below the horizon.

Cosmos exposures were made with detectors mounted in one of three possible locations on the satellite's spherical exterior, corresponding to three different attitudes in passing through the radiation belts. The two exposures shown in Figure 2 came from one of these attitudes. In Figure 3, we show distributions of the azimuth angle $\phi$ from six different Cosmos exposures, including an SEP event and a quiet-time exposure in each detector attitude. Striking differences between SEP and quiet-time exposures are seen in all three attitudes. Apart from obstructed arrival directions, the observed SEP distributions are flat. In the quiet-time exposures, however, the fluxes are highly anisotropic, with strong azimuthal variation.

\section{Monte Carlo Simulations}

The angular distributions in Figures 2 and 3 strongly suggest a trapped-particle component in the Cosmos data [Grigorov et al., 1990]. We tested this interpretation with detailed Monte Carlo simulations of the exposures; as shown in Figure 3. The SEP simulations ensure that the obstructions are well understood. The quiet-time simulations show that a trapped-particle component gives an internally consistent view of the data, by accounting for the differences among the three detector attitudes.

To carry out the simulations we used tracking data to reconstruct the orbital trajectory of each flight on a secondby-second basis. At each location along the trajectory, the simulation sampled from a model of the incident flux to specify a particle's velocity vector and energy. The Monte Carlo program then accounted for losses due to obstructions, some small detection inefficiencies, and the obliquity factor. For each Cosmos flight we simulated the expected distributions of both cosmic rays and trapped particles. We then minimized $\chi^{2}$ to fit the observed azimuth distribution to a linear combination of cosmic-ray and trapped components.

In simulating SEPs and quiet-time cosmic rays, the flux was assumed to be isotropic except for directions below the Earth's horizon, where the flux was set to zero. (Cosmic-ray anisotropies, such as the east-west effect, and penumbral effects are small throughout the range of particle rigidities involved here.) Particle energies were sampled from orbitaveraged spectra, as determined from contemporaneous flux measurements from IMP-8 convolved with the geomagnetic transmission function [cf. Adams et al., 1991a].

To simulate trapped particles, we constructed a simple model based on the fact that trapped ions are observable at Cosmos altitudes only near their mirror points in the South Atlantic Anomaly (SAA). Both anomalous-component trapping [Blake and Friesen, 1977] and radial diffusion of ions from the outer zone [Panasyuk, 1984] predict that trapped oxygen ions of these energies should be found primarily at $L>2$. The simulations therefore restricted the trapped flux to the portion of the SAA at $2<\mathrm{L}<3$. The intensity and spectrum of the trapped particles were assumed to be the same everywhere within this region. In simulating trapped-particle velocity vectors $(v)$, the local pitch angle distribution was approximated by sampling the angle between $v$ and the local magnetic field vector $B$ (as calculated from IGRF 1985 [Barraclough et al., 1987]) from a Gaussian centered at $90^{\circ}$ with standard deviation $6^{\circ}$. The 
Isolropic Solar Energetic Particles Isotropic Cosmle Rays + Trapped Particles
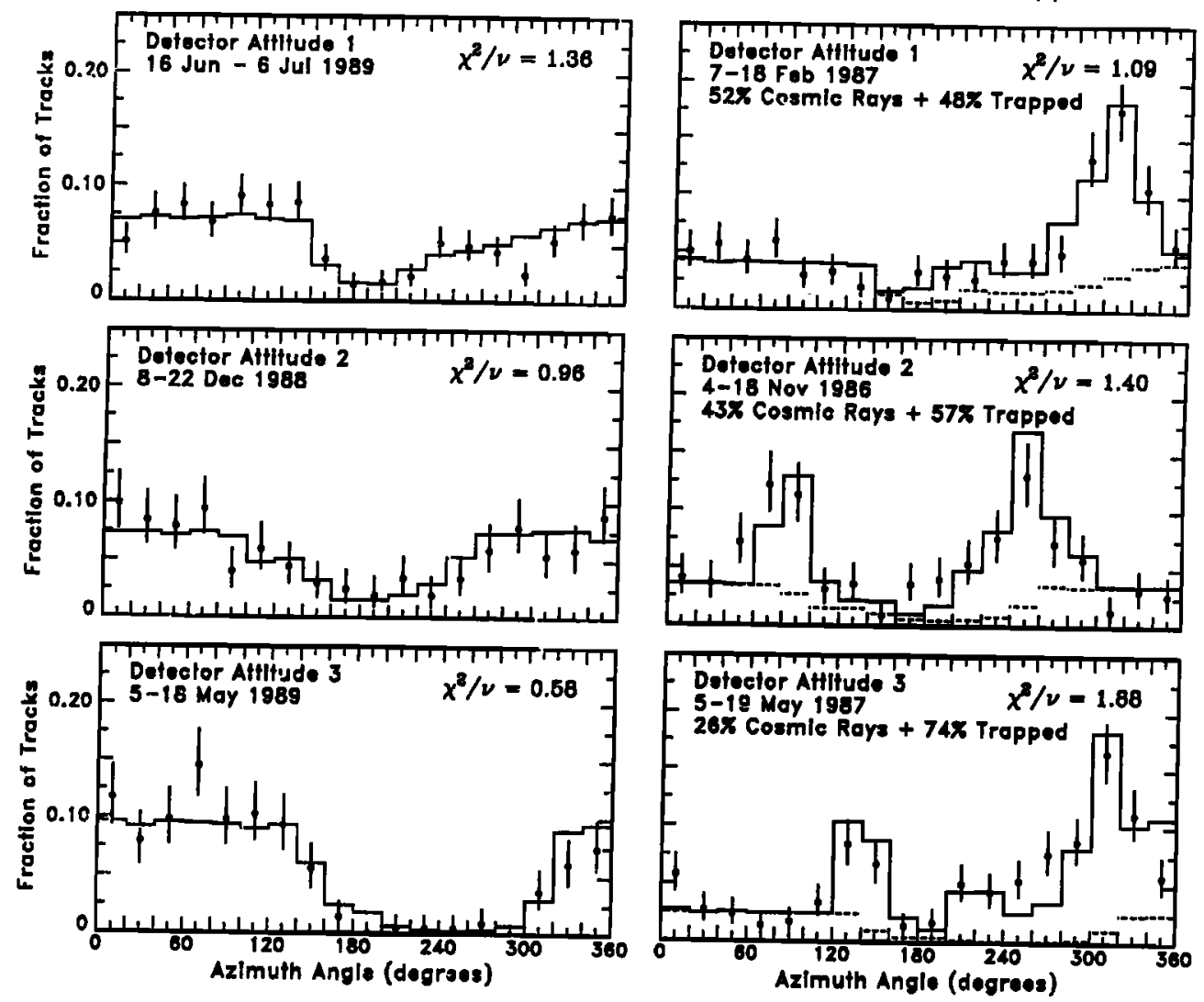

Fig. 3. Distributions of the azimuth angle $\phi$ for SEP exposures (left) and quiet-time exposures (right) for three different detector attitudes. The points are the Cosmos data with statistical error bars, and the histograms are Monte Carlo simulations (see text for details). In the panels on the right, the dashed lines show the simulated cosmic-ray contribution and the solid lines show the sum of simulated cosmic-ray and trapped components. Statistical errors on the simulations are negligible compared to those of the data. The dates for each exposure, the reduced $\chi^{2}$ of the simulations' fits to the data, and the best-fit percentages of cosmic rays and trapped particles in the quiet-time exposures are also shown. The bottom two panels show the same data as in Figure 2.

component of $\mathbf{v}$ perpendicular to $\mathbf{B}$ was then randomly oriented. Trapped-particle energies were sampled from a relatively flat spectrum, as suggested by models of both anomalous-component trapping [Blake, 1990] and radial diffusion [Spjeldvik and Fritz, 1978].

We used the simulations to check the assumed geomagnetic distribution of trapped particles. In addition to placing the trapped flux at $2<\mathrm{L}<3$, we also ran simulations in which trapped oxygen particles were assumed throughout the SAA or only at $1.2<\mathrm{L}<1.5$ (where the trapped proton flux is most intense at Cosmos altitudes). In these cases, the simulated azimuthal accumulations shifted so as to produce significantly poorer agreement with the data.

The agreement between the data and simulations in Figure 3 is generally good, thus confirming that the quiettime azimuthal accumulations are due to trapped particles near their mirror points. Some discrepancies remain, but this is not unexpected given the simplicity of our trapped particle model. In addition to azimuth distributions, we also compared the observed and simulated distributions of dip angles and particle ranges in the detector. We also checked the correlations among the $\theta, \phi$, and range observables. In all cases there was satisfactory agreement with the data.

\section{Origin of the Trapped Particles}

We have considered two possible origins for these particles. The first is trapping of anomalous cosmic-ray ions [Blake and Friesen, 1977]. All of our observations, including geomagnetic location, spectrum, composition, and temporal variation, appear consistent with this explanation. A second possible origin is radial diffusion from the outer radiation belt. This explanation, however, appears inconsistent with the data in two ways. First, standard radial diffusion theory is unable to account for the acceleration of ionospheric ions to the energies observed here. Outer-zone ions with the necessary initial energies must therefore be of solar wind or (more likely) of SEP origin. For these sources, the $\mathrm{C} / \mathrm{O}$ ratio is typically $\sim 0.5$. The observed $\mathrm{C} / \mathrm{O}$ ratio in the Cosmos data, however, is $\leqslant$ a few percent, consistent with the observed composition of anomalous cosmic rays at this time [cf. Adams et al., 1991a]. It is difficult to understand how radial diffusion could produce such a large change in particle composition. Second, the trapped flux is variable and anticorrelated with solar activity. Figure 4 compares the time history of the measured trapped-oxygen flux near the mirror point, $J_{\perp}$, with the IMP-8 quiet-time 


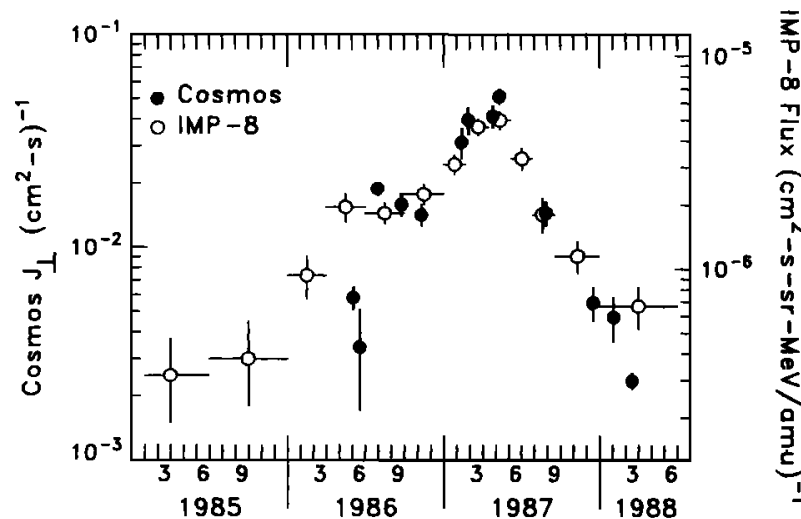

Fig. 4. Time history of the trapped flux $J_{\perp}$ (left-hand scale) measured on the Cosmos flights and of the quiet-time 5-11 MeV/nucleon oxygen flux (right-hand scale) measured by the Caltech instrument on IMP-8. The IMP-8 fluxes are dominated by the anomalous component and uncorrected for contributions from Galactic cosmic rays and solar particles, which are small $(<10 \%)$ except at low flux levels.

measurements of the anomalous-component oxygen flux in interplanetary space. The temporal variations are very similar, and both the anomalous component and the trapped flux reached their peaks near solar minimum in early 1987. Because radial diffusion is promoted by large magnetic storms caused by solar flares, it is unclear why a trapped flux from this process would peak at solar minimum.

In future work we will search for other trapped heavy ions species. We will also build a detailed dynamical model of anomalous-component trapping which may be tested with the Cosmos data.

In summary, comparing the Cosmos data with our simulated distributions clearly demonstrates the presence of trapped energetic oxygen ions in the inner magnetosphere. The characteristics of this trapped flux are fully consistent with originating from the anomalous component of cosmic rays. This work also demonstrates that passive track detectors flown aboard 3-axis stabilized spacecraft can be a powerful tool for studying trapped particles. With appropriate selection criteria on particle arrival direction, a sample of trapped particles with a small, calculable background of cosmic rays can be isolated. Since track detectors with large collecting area can be inexpensively manufactured and flown, this method can be used to explore even rare components of the trapped radiation.

Acknowledgments. We gratefully acknowledge the efforts of NASA and IKI in the US/USSR Joint Working Group on Solar-Terrestrial Physics, which sponsored this study. We especially thank Vernon Jones for his encouragement and support. We thank D.I. Kozlov, general contractor for Cosmos, for the many flights that provided the Cosmos data. We also thank the Space Analysis and Data Branch (J3SOS) of the US Space Command for providing the Cosmos orbital data. This work is supported in part by NASA contract W-17,358, by NASA grant NAG5-727, and by the Aerospace Sponsored Research (ASR) Program of the Aerospace Corporation.

\section{References}

Adams, J.H., Jr. et al., The charge state of the anomalous component, Astrophys. J. Lett., 375, L45-L48, 1991 a.

Adams, J.H., Jr., L.P. Beahm, and A.J. Tylka, The charge state of the anomalous component: Results from the Trapped Ions in Space experiment, Astrophys. J., 377, 292-305, 1991b.

Barraclough, D.R. et al., International Geomagnetic Reference Field Revision 1987, J. Geomag. Geoelectr., 39, 773-779, 1987.

Biswas, S., and N. Durgaprasad, Skylab measurements of low energy cosmic rays, Space Sci. Rev., 25, 285-327, 1980.

Blake, J.B., Geomagnetically trapped heavy ions from anomalous cosmic rays, Proc. 21st Intl. Cosmic Ray Conf., 7, 30-33, 1990.

Blake, J.B., and L.M. Friesen, A technique to determine the charge state of the anomalous low-energy cosmic rays, Proc. 15th Intl. Cosmic Ray Conf., 2, 341-346, 1977.

Chan, J.H., and P.B. Price, Composition and energy spectra of heavy nuclei of unknown origin detected on Skylab, Phys. Rev. Lett., 35, 539-542, 1975.

Fisk, L.A., B. Kozlovsky, and R. Ramaty, An interpretation of the observed oxygen and nitrogen enhancements in low-energy cosmic rays, Astrophys. J. Lett., 190, L35L37, 1974.

Grigorov, N.L. et al., The charge state of anomalous oxygen of cosmic rays, Geomag. and Aeron., 29, 889-891, 1989.

Grigorov, N.L. et al., Anomalous oxygen in the near-Earth space in 1985-88, Proc. 21st Intl. Cosmic Ray Conf., 6, 176-179, 1990.

Mogro-Campero, A., and J.A. Simpson, Identification and relative abundances of $\mathrm{C}, \mathrm{N}$, and $\mathrm{O}$ nuclei trapped in the geomagnetic field, Phys. Rev. Lett., 25, 1631-1634, 1970.

Oschlies, K., R. Beaujean, and W. Enge, On the charge state of anomalous oxygen, Astrophys. J., 345, 776-781, 1989.

Panasyuk, M.I., Experimental verification of ion transport mechanisms in the radiation belts of the Earth under the effect of fluctuating electric fields, Cosmic Res., 22, 468482, 1984.

Pesses, M.E., J.R. Jokipii, and D. Eichler, Cosmic ray drift, shock wave acceleration, and the anomalous component of cosmic rays, Astrophys. J. Lett., 246, L85-L89, 1981.

Spjeldvik, W.N., and T.A. Fritz, Theory for charge states of energetic oxygen ions in the Earth's radiation belts, $J$. Geophys. Res., 83, 1583-1598, 1978.

Webber, W.R., Composition of anomalous cosmic rays, $A I P$ Conf. Proc., 183, 100-110, 1989.

N.L. Grigorov, M.A. Kondratyeva, M.I. Panasyuk, and Ch.A. Tretyakova, Institute for Nuclear Physics, Moscow State University, Moscow 119899 USSR.

J.H. Adams, Jr. and A.J. Tylka, Code 4154, Naval

Research Laboratory, Washington, DC 20375-5000 USA.

J.B. Blake and M. Schulz, The Aerospace Corporation, P.O. Box 92957, Los Angeles, CA 90009 USA.

R.A. Mewaldt, 220-47 Downs Laboratory, California Institute of Technology, Pasadena, CA 91125 USA.

(Received: August 14, 1991; accepted September 9, 1991.) 